\title{
Improved statistical model on the effect of random errors in the phase and amplitude of element excitations on the array radiation pattern
}

Citation for published version (APA):

van den Biggelaar, A. J., Johannsen, U., Mattheijssen, P., \& Smolders, A. B. (2018). Improved statistical model on the effect of random errors in the phase and amplitude of element excitations on the array radiation pattern. IEEE Transactions on Antennas and Propagation, 66(5), 2309-2317. https://doi.org/10.1109/TAP.2018.2800519

DOI:

10.1109/TAP.2018.2800519

Document status and date:

Published: 01/05/2018

Document Version:

Accepted manuscript including changes made at the peer-review stage

Please check the document version of this publication:

- A submitted manuscript is the version of the article upon submission and before peer-review. There can be important differences between the submitted version and the official published version of record. People interested in the research are advised to contact the author for the final version of the publication, or visit the DOI to the publisher's website.

- The final author version and the galley proof are versions of the publication after peer review.

- The final published version features the final layout of the paper including the volume, issue and page numbers.

Link to publication

\footnotetext{
General rights

- You may freely distribute the URL identifying the publication in the public portal. follow below link for the End User Agreement:

www.tue.nl/taverne

Take down policy

If you believe that this document breaches copyright please contact us at:

openaccess@tue.nl

providing details and we will investigate your claim.
}

Copyright and moral rights for the publications made accessible in the public portal are retained by the authors and/or other copyright owners and it is a condition of accessing publications that users recognise and abide by the legal requirements associated with these rights.

- Users may download and print one copy of any publication from the public portal for the purpose of private study or research.

- You may not further distribute the material or use it for any profit-making activity or commercial gain

If the publication is distributed under the terms of Article $25 \mathrm{fa}$ of the Dutch Copyright Act, indicated by the "Taverne" license above, please 


\title{
Improved Statistical Model on the Effect of Random Errors in the Phase and Amplitude of Element Excitations on the Array Radiation Pattern
}

\author{
A.J. van den Biggelaar, U. Johannsen, P. Mattheijssen, A.B. Smolders
}

\begin{abstract}
Due to errors in the phase and amplitude of element excitations in an antenna array, the array radiation pattern gets distorted. In order to derive statistical results on this distortion, specifically on determining the probability of exceeding a certain side lobe level, it is often assumed that the magnitude of the array amplitude pattern follows a Rician distribution. It is shown that the Rician distribution implies two assumptions and, therefore, a more general Beckmann distribution is proposed to describe the distribution of the magnitude of the array amplitude pattern. Using Monte Carlo simulations, it is shown that the use of a Beckmann distribution outperforms the Rician distribution. Also, the seemingly counterintuitive result, that the maximum probability of exceeding a certain side lobe level, in general, does not have to be at the angle where the highest side lobe in the error-free case is located, is obtained. Due to this result, the importance of using an angular probability plot is emphasized. Furthermore, a physical explanation for the observed seemingly counterintuitive behavior is given.
\end{abstract}

Index Terms-Antenna radiation patterns, error analysis, phased arrays, probability, random noise.

\section{INTRODUCTION}

The next generation radio access networks, often called 5G, will make use of beam steering techniques to improve the data throughput to mobile users. To realize a beam steering radio, active phased arrays will be used. In order to reduce interference to other users, the side lobe level (SLL) needs to be well controlled. It is a straightforward task to determine the proper element excitations to get a desired array radiation pattern using standard synthesis techniques. However, due to errors in the phase and amplitude of the element excitations, the array radiation pattern gets distorted. This distortion can result in an array radiation pattern having a higher SLL than initially designed for. Therefore, knowledge about the impact of these errors on the array radiation pattern, specifically the SLL, is of high importance for future $5 \mathrm{G}$ networks. These errors can, in general, be divided in two types; systematic/predictable and random/unpredictable errors. In the past, a lot of effort has already been done in order to derive statistical results on the effect of the array radiation pattern due to the random errors. In fact, in 1952 the first statistical results on distorted array radiation patterns were already obtained by Ruze [1].

In [1], the claim has been made that the distribution of the magnitude of the array amplitude pattern follows a modified Rayleigh (nowadays commonly known as Rician) distribution. Many publications addressing this problem followed, see e.g. [2-7], and this Rician distribution is used in all these publications to model the distribution of the magnitude of the array amplitude pattern. However, as presented in this paper, in some cases the use of the Rician distribution is not sufficient. Therefore, the Beckmann distribution is proposed to address this problem and it is shown, that the usage of the proposed distribution outperforms the usage of the conventionally used Rician distribution. Using the Beckmann distribution, also a seemingly counterintuitive result is obtained, i.e. that the maximum probability of exceeding a certain SLL does not have to be at the position(s) of the highest side lobe(s). Therefore, angular probability plots are introduced, which cover the whole angular domain, rather than only looking at the highest side lobe(s).

The outline of this paper is as follows. In Section II, the random errors and the random variables for the real and imaginary values of the distorted array amplitude pattern are introduced. In Section III statistical quantities of these random variables are presented. Section IV is devoted to choosing the proper distribution for the array amplitude pattern. Here, both the Rician and Beckmann distribution are discussed. Section V introduces the angular probability plots and compares the Rician and Beckmann cumulative distribution functions (CDFs) with Monte Carlo simulations. Here, it is shown that the use of the Beckmann CDF yields more accurate results compared to the usage of the Rician CDF. Section VI is devoted to a physical interpretation of the obtained results. Finally, in Section VII, conclusions and future work is presented.

\section{THE ARRAY AMPLITUDE PATTERN IN THE PRESENCE OF RANDOM ERRORS}

For a one-dimensional linear antenna array, the complex-valued array amplitude pattern $F_{0}(\theta)$ (sometimes also called array field or voltage pattern) in the azimuth plane can be expressed as

$$
F_{0}(\theta)=\sum_{n=1}^{N} g_{n}(\theta) a_{n} e^{j k_{0} d(N-n) \sin \theta} .
$$

Here, $N$ is the number of antenna elements, $g_{n}(\theta)$ and $a_{n}$ are the real-valued element pattern and the complex-valued excitation coefficient of the $n^{\text {th }}$ antenna element, respectively, $k_{0}$ is the wave number in vacuum and $d$ is the spacing between the individual antenna elements. The array amplitude pattern as given in (1) can be split in a real and imaginary field component using Euler's formula, i.e.,

$$
\begin{aligned}
& \operatorname{Re}\left[F_{0}(\theta)\right]=\sum_{n=1}^{N} A_{n}(\theta) \operatorname{Re}\left[e^{j C_{n}(\theta)}\right]=\sum_{n=1}^{N} A_{n}(\theta) \cos C_{n}(\theta), \\
& \operatorname{Im}\left[F_{0}(\theta)\right]=\sum_{n=1}^{N} A_{n}(\theta) \operatorname{Im}\left[e^{j C_{n}(\theta)}\right]=\sum_{n=1}^{N} A_{n}(\theta) \sin C_{n}(\theta),
\end{aligned}
$$

where,

$$
\begin{aligned}
& A_{n}(\theta)=g_{n}(\theta)\left|a_{n}\right|, \\
& C_{n}(\theta)=k_{0} d(N-n) \sin \theta+\arg \left(a_{n}\right) .
\end{aligned}
$$

Here, $\arg ($.$) represents the function to calculate the argument or$ phase of a complex number. In the remainder of the paper, the notation of $F_{0}(\theta), g_{n}(\theta), A_{n}(\theta)$ and $C_{n}(\theta)$ is shortened by omitting the explicit mentioning of the $\theta$-dependency.

The phase and amplitude of the element excitations are subject to nondeterministic noise and errors and, therefore, two random variables are introduced for each individual element. The random 
variables $\delta_{p n}$ and $\delta_{a n}$ represent the phase and relative amplitude error of the $n^{\text {th }}$ antenna element, respectively. Both random variables are Gaussian distributed and have zero mean, but, in general, have a different variance. However, the variance in the phase error is assumed to be identical amongst the different elements. The same holds for the variance of the amplitude error. Mathematically this can be expressed as

$$
\begin{aligned}
& \delta_{p n} \sim \mathcal{N}\left(0, \sigma_{p}^{2}\right), \\
& \delta_{a n} \sim \mathcal{N}\left(0, \sigma_{a}^{2}\right), \\
& \text { where } n=1, \ldots, N,
\end{aligned}
$$

with $\sigma_{p}^{2}$ and $\sigma_{a}^{2}$ the variance of the phase and relative amplitude error, respectively. Furthermore, no correlation between errors is assumed, i.e.,

$$
\begin{aligned}
& \operatorname{corr}\left(\delta_{p n}, \delta_{p m}\right)=0, \text { for } n \neq m, \\
& \operatorname{corr}\left(\delta_{a n}, \delta_{a m}\right)=0, \text { for } n \neq m, \\
& \operatorname{corr}\left(\delta_{a n}, \delta_{p m}\right)=0, \text { for all } n, m, \\
& \text { where } n=1, \ldots, N, \text { and } m=1, \ldots, N .
\end{aligned}
$$

The error-free array amplitude pattern $F_{0}$ will be distorted as a result of the introduced random errors. The distorted array amplitude pattern $F_{d}$ can be written as

$$
F_{d}=\sum_{n=1}^{N} A_{n}\left(1+\delta_{a n}\right) e^{j \delta_{p n}} e^{j C_{n}} .
$$

Again, this distorted array amplitude pattern can be split in a real and imaginary component using Euler's formula, i.e.,

$$
\begin{aligned}
& X=\operatorname{Re}\left[F_{d}\right]=\sum_{n=1}^{N} A_{n}\left(1+\delta_{a n}\right)\left(\cos \delta_{p n} \cos C_{n}-\sin \delta_{p n} \sin C_{n}\right), \\
& Y=\operatorname{Im}\left[F_{d}\right]=\sum_{n=1}^{N} A_{n}\left(1+\delta_{a n}\right)\left(\cos \delta_{p n} \sin C_{n}+\sin \delta_{p n} \cos C_{n}\right) .
\end{aligned}
$$

\section{STATISTICAL QUANTITIES OF THE ARRAY AMPLITUDE PATTERN}

According to the Central Limit Theorem (CLT), for sufficiently large $N$, the random variables $X$ and $Y$ will follow a Gaussian distribution. In the following subsections, some statistical quantities of these Gaussian distributions are presented. ${ }^{1}$

\section{A. Mean value}

The mean values of $X$ and $Y$ can be written as

$$
\begin{aligned}
& E[X]=\mu_{x}=e^{-\frac{\sigma_{p}^{2}}{2}} \operatorname{Re}\left[F_{0}\right], \\
& E[Y]=\mu_{y}=e^{-\frac{\sigma_{p}^{2}}{2}} \operatorname{Im}\left[F_{0}\right] .
\end{aligned}
$$

${ }^{1}$ For a complete derivation of these statistical quantities, please send an e-mail to the main author.

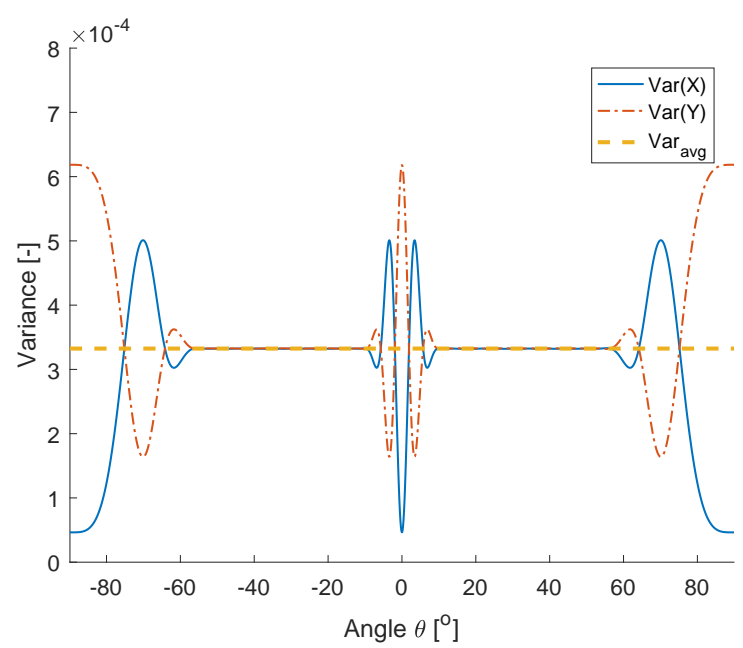

Fig. 1: Variance of $X, Y$ and its average. Parameters as given in Section IIIb.

\section{B. Variance}

The variances of $X$ and $Y$ can be written as

$$
\begin{aligned}
\operatorname{Var}(X)=\sigma_{x}^{2}= & \frac{1}{2}\left(1+\sigma_{a}^{2}\right)\left(1-e^{-2 \sigma_{p}^{2}}\right) \sum_{n=1}^{N} A_{n}^{2} \\
& +\left(\left(1+\sigma_{a}^{2}\right) e^{-2 \sigma_{p}^{2}}-e^{-\sigma_{p}^{2}}\right) \sum_{n=1}^{N} A_{n}^{2} \cos ^{2} C_{n}
\end{aligned}
$$

$$
\begin{aligned}
\operatorname{Var}(Y)=\sigma_{y}^{2}= & \frac{1}{2}\left(1+\sigma_{a}^{2}\right)\left(1-e^{-2 \sigma_{p}^{2}}\right) \sum_{n=1}^{N} A_{n}^{2} \\
& +\left(\left(1+\sigma_{a}^{2}\right) e^{-2 \sigma_{p}^{2}}-e^{-\sigma_{p}^{2}}\right) \sum_{n=1}^{N} A_{n}^{2} \sin ^{2} C_{n}
\end{aligned}
$$

Please note that $C_{n}$ has a $\theta$-dependency (see (3)), and therefore the variance of $X$ and $Y$ are dependent on $\theta$ as well. In Fig. 1, this dependency is shown. To generate this result, and the results in the subsequent sections, an array of $N=16$ elements with halfwavelength spacing $d=\frac{1}{2} \lambda_{0}$ has been chosen. Chebyshev tapering with a designed SLL of $S L L_{0}=-40 \mathrm{~dB}$ has been applied to this array. The standard deviation of the phase and amplitude errors were chosen to be $\sigma_{p}=5^{\circ}$ and $\sigma_{a}=0.1 \mathrm{~dB}$, respectively. Furthermore, the amplitude pattern of the individual elements $g_{n}$ is isotropic (i.e., $g_{n}=1$ ), unless otherwise specified. The following derived results and drawn conclusion are in principle, however, valid for other sets of parameters as well.

Note that in Fig. 1, around $\theta=-90^{\circ}, \theta=0^{\circ}$ and $\theta=90^{\circ}$, the variances show large variations. In Section VI, a physical interpretation for this behavior is presented.

\section{Correlation}

The correlation coefficient $\rho_{x y}$ between random variables $X$ and $Y$ with expected values $\mu_{x}$ and $\mu_{y}$ and standard deviations $\sigma_{x}$ and $\sigma_{y}$ is defined as

$$
\rho_{x y}=\frac{\operatorname{cov}(X, Y)}{\sigma_{x} \sigma_{y}}=\frac{\operatorname{cov}(X, Y)}{\sqrt{\operatorname{Var}(X) \operatorname{Var}(Y)}} .
$$

The covariance between the random variables $X$ and $Y$ can be written as 


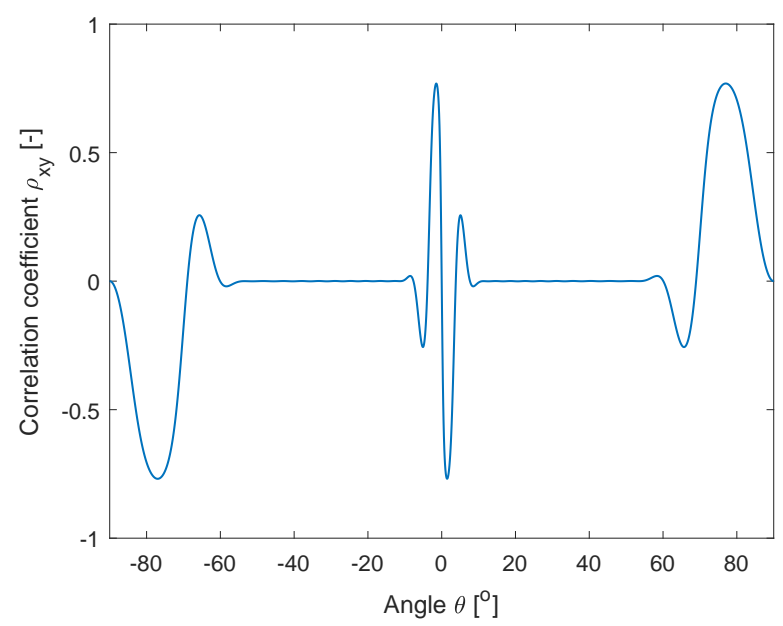

Fig. 2: Correlation between $X$ and $Y$. Parameters as given in Section IIIb.

$$
\operatorname{cov}(X, Y)=\left(\left(1+\sigma_{a}^{2}\right) e^{-2 \sigma_{p}^{2}}-e^{-\sigma_{p}^{2}}\right) \sum_{n=1}^{N} A_{n}^{2} \cos C_{n} \sin C_{n}
$$

By substituting (10), (11) and (13) into (12), an expression for the correlation coefficient $\rho_{x y}$ can be found. This will give rise to a tedious expression and therefore this expression has been omitted in this paper. However, the reader is able to compute the correlation coefficient $\rho_{x y}$ using the equations presented in this section.

In Fig. 2, the correlation between $X$ and $Y$ is shown as function of $\theta$ for the parameters as specified in Section IIIb. As can be seen in Fig. 2, there is a strong correlation at certain angles. This means that although the phase and relative amplitude errors are uncorrelated, the real and imaginary value of the distorted array amplitude pattern can, in general, be highly correlated at certain angles. In Section VI, a physical interpretation for this observed correlation is presented.

\section{Statistics ON THE MAGNitUde OF THE ARRAY AMPLITUDE PATTERN}

In the previous section, statistical quantities are presented which allow the calculation of the distribution of the real and imaginary value of the array amplitude pattern. From a designer's perspective, however, it is far more interesting to be able to calculate the distribution of the magnitude of the array amplitude pattern. It is often assumed that the magnitude of the array amplitude pattern follows a Rician distribution. However, by using the Rician distribution, two assumptions are implicitly made and therefore a Beckmann distribution is proposed in this paper. In this section, both distributions are discussed and compared.

\section{A. Rician distribution}

Supposing there are two orthogonal and uncorrelated Gaussian distributions. If these Gaussian distributions have a nonzero and unidentical mean $\mu_{x}$ and $\mu_{y}$, but share the same variance $\sigma^{2}$, then the magnitude $r$ of the sum of these Gaussian distributions will follow a Rician distribution. The Rician probability density function (PDF) is defined as

$$
P D F_{\text {rice }}(r)=\frac{r}{\sigma^{2}} \exp \left(-\frac{r^{2}+\nu^{2}}{2 \sigma^{2}}\right) I_{0}\left(\frac{r \nu}{\sigma^{2}}\right) .
$$

Here, $I_{0}$ is the modified Bessel function of the first kind with order zero and $\nu$ is defined as

$$
\nu=\sqrt{\mu_{x}^{2}+\mu_{y}^{2}} .
$$

The CDF of a Rician distribution is given by

$$
C D F_{\text {rice }}(r)=1-Q_{1}\left(\frac{\nu}{\sigma}, \frac{r}{\sigma}\right)
$$

with $Q_{1}$ the Marcum Q-function [8].

This Rician PDF is often used to describe the distribution of the magnitude of the array amplitude pattern. However, by using the Rician distribution, two assumptions on the statistic quantities of the random variables $X$ and $Y$ are implicitly made; the variances of $X$ and $Y$ are identical, and the correlation between $X$ and $Y$ has to be zero. In [1], the variances of $X$ and $Y$ are averaged and zero correlation between $X$ and $Y$ is assumed. [4-7] follow the result found in [1]. In [2,3], the authors come to the same expressions for the variances and correlation as presented in Section III. However, when assessing the distribution of the magnitude of the array amplitude pattern, the same assumptions on the variances and correlation as in [1] are made.

Following the approach in [1] and averaging the variance found in (10) and (11) yields

$$
\operatorname{Var}_{a v g}=\frac{\operatorname{Var}(X)+\operatorname{Var}(Y)}{2}=\frac{1}{2}\left(1+\sigma_{a}^{2}-e^{-\sigma_{p}^{2}}\right) \sum_{n=1}^{N} A_{n}^{2}
$$

As can be seen in (17), the averaged variance is constant with respect to $\theta$. In Fig. 1, the averaged variance is compared to the variance of $X$ and $Y$ for the parameters as specified in Section IIIb. Hence, if the designer is interested in the maximum probability of exceeding a specified SLL, the Rician CDF only has to be calculated at the angle with the highest value of $\nu$ to obtain the maximum probability of exceeding a specified SLL. This turns out to be at the position(s) of the highest side lobe(s).

\section{B. Beckmann distribution}

The Beckmann distribution is a more general distribution compared to the Rician distribution. Again, supposing there are two orthogonal Gaussian distributions, but in this case these distributions can be correlated. These Gaussian distributions can have a nonzero and unidentical mean $\mu_{x}, \mu_{y}$ and variance $\sigma_{x}^{2}, \sigma_{y}^{2}$. Then, the magnitude $r$ of the sum of these Gaussian distributions will follow a Beckmann distribution. Note that for the case that $\sigma_{x}^{2}=\sigma_{y}^{2}$ and $\rho_{x y}=0$, the Beckmann distribution reduces to a Rician distribution.

The Beckmann PDF is defined by [9]

$$
P D F_{b e c k}(r)=\frac{r}{2 \pi \sigma_{x} \sigma_{y} \sqrt{1-\rho_{x y}^{2}}} \int_{0}^{2 \pi} \exp \left(-\frac{z}{1-\rho_{x y}^{2}}\right) d \theta,
$$

with

$$
\begin{aligned}
z= & \frac{\left(r \cos \theta-\mu_{x}\right)^{2}}{2 \sigma_{x}^{2}}+\frac{\left(r \sin \theta-\mu_{y}\right)^{2}}{2 \sigma_{y}^{2}} \\
& -\frac{\rho_{x y}\left(r \cos \theta-\mu_{x}\right)\left(r \sin \theta-\mu_{y}\right)}{\sigma_{x} \sigma_{y}} .
\end{aligned}
$$

The CDF can then be calculated by

$$
C D F_{b e c k}(r)=\int_{0}^{r} P D F_{b e c k}\left(r^{\prime}\right) d r^{\prime} .
$$

Equation (20) unfortunately does not have a closed-form solution and, therefore, has to be calculated numerically. 


\section{REsults}

To verify that the magnitude of the array amplitude pattern follows a Beckmann distribution, the CDF of (20) is compared to Monte Carlo simulations. For each Monte Carlo simulation, a phase and amplitude error is generated for each element according to the probability distributions as defined in (4), where $\sigma_{p}=5^{\circ}$ and $\sigma_{a}=0.1 \mathrm{~dB}$. The resulting array power pattern is calculated and for each simulation, as function of angle, is determined whether a certain desired side lobe level $S L L_{e}[\mathrm{~dB}]$, with respect to the error-free case, is exceeded. In Fig. 3, an example of this process is shown. Here, the errorfree array radiation pattern and one of the calculated distorted array radiation patterns is shown. At $53^{\circ}<\theta<59^{\circ}$, the distorted pattern exceeds $S L L_{e}$. For these angles the value 1 is assigned, whereas for the other angles the value 0 is assigned. Averaging these values over all simulations leads to an estimate of the probability of exceeding $S L L_{e}$ as function of angle. This estimate is compared to the probability determined by the Rician and Beckmann CDF. In the upcoming angular probability plots, the desired side lobe level is set to $S L L_{e}=-25 \mathrm{~dB}$ and the Monte Carlo simulation curve is the determined probability as a result of 1 million simulations.

Figure 4 shows an angular probability plot with the parameters as described in Section IIIb. The main lobe can, in general, be discarded from the analysis and is illustrated by a gray area. The Monte Carlo simulation is compared to the results of the conventional Rician CDF and the proposed Beckmann CDF. One can see that the 3 methods agree for $|\theta|<60^{\circ}$. For $|\theta|>60^{\circ}$, the calculated values of the Rician CDF starts to show a probability mismatch compared to the calculated values of the Beckmann CDF and Monte Carlo simulations. The Beckmann CDF, however, still agrees with the Monte Carlo simulations over the whole angular domain.

The behavior of the Monte Carlo simulations and Beckmann CDF beyond $|\theta|=60^{\circ}$ may seem counterintuitive. Although the side lobes of the error-free pattern have equal amplitudes (see Fig. 3), the most outward side lobes dominate in this case the probability of exceeding a certain radiation level. A physical interpretation of this behavior is given in Section VI.

One may argue that for a lot of practical antennas, the radiation pattern of the individual elements used in antenna arrays hardly have radiation in the end-fire directions, and therefore the mismatch found in Fig. 4 is not of high importance. However, in Fig. 5, the same angular probability plot is shown, but now a linear phase distribution is applied, such that the beam is steered to $45^{\circ}$. Here, one can see that the probability mismatch is shifted to the region $-25^{\circ}<\theta<$ $-10^{\circ}$. The question might arise why this shift occurs and whether the position of the probability mismatch is predictable. In Section VI,

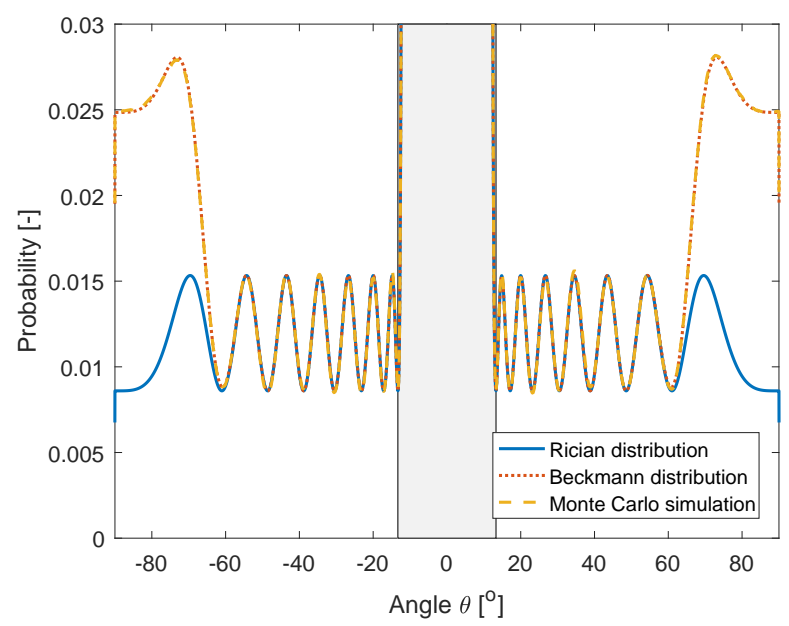

Fig. 4: Angular probability plot. For $|\theta|>60^{\circ}$, the Rician CDF starts to deviate from the Beckmann CDF and Monte Carlo simulation.

insight in this shift is given and a method is presented which enables the prediction of the location of this increase in probability.

To illustrate that this sudden increase in probability can impose a problem for a practical antenna array, a configuration with a different individual radiation pattern has been chosen. In this case, the power pattern of the individual elements follows a cosine shape, i.e., $g_{n}^{2}(\theta)=\cos (\theta)$ for $n=1, \ldots, N$. In Fig. 6 the result is shown. Again, the beam is steered to $45^{\circ}$ using a linear phase distribution.

A few things have to be noted here. Chebyshev tapering does not take into account the cosine shaped radiation pattern of the individual elements. Therefore, the side lobes of the error-free radiation pattern will not be constant. Instead, the envelope of the array radiation pattern will follow a cosine shape, as can be observed in Fig. 7. Due to this, the envelope of the biggest part of the probability curve in Fig. 6 also follows a cosine shape. Moreover, the overall probability of exceeding the threshold value is increased compared to the case where only uniform radiators were being used. Furthermore, by properly inspecting Fig. 6 and 7, it can be seen that the highest probability of exceeding $S L L_{e}$ is not at the location of the largest side lobe in the error-free case. Finally, for such a practical setting, the probability mismatch between the Rician CDF and the Monte Carlo simulations is large, making the usage of the Beckmann CDF preferable compared to the Rician CDF.

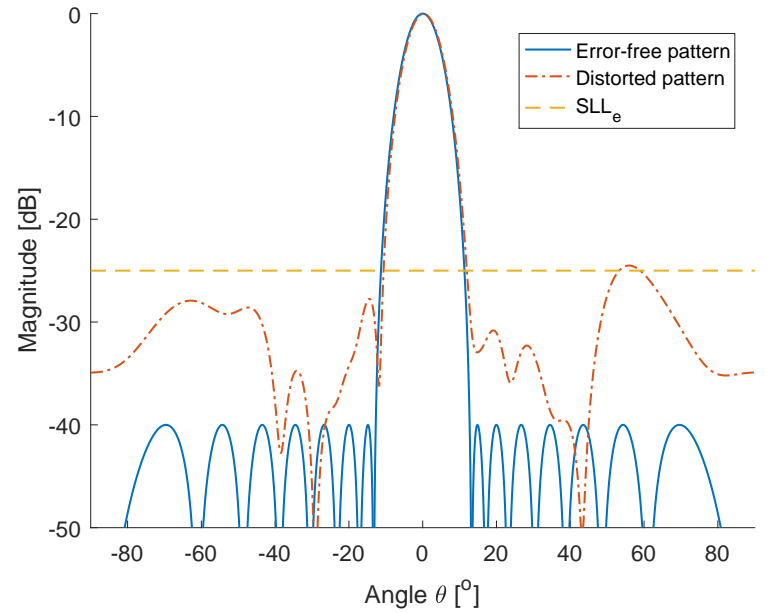

Fig. 3: Example of one out of million Monte Carlo simulations. Around $53^{\circ}<\theta<$ $59^{\circ}$, the distorted pattern exceeds $S L L_{e}$

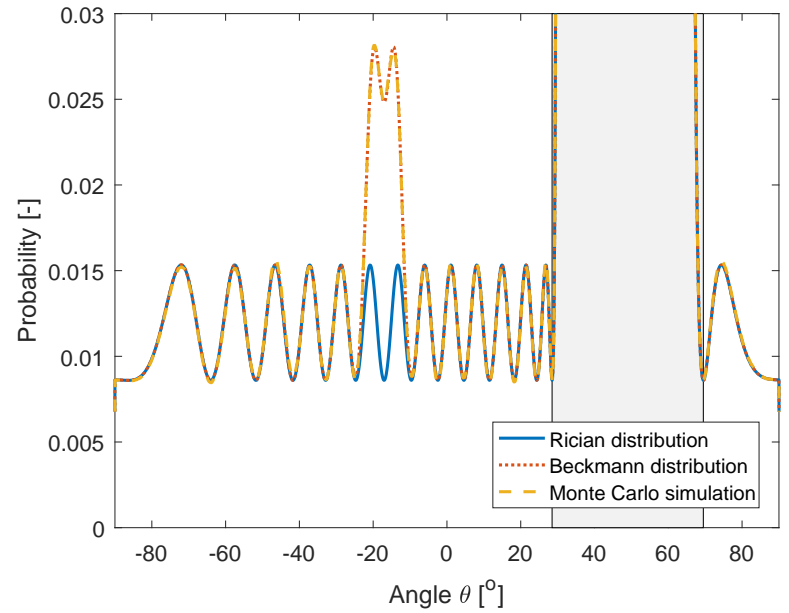

Fig. 5: Angular probability plot with a steering angle of $45^{\circ}$. The mismatch in probability is shifted to the region $-25^{\circ}<\theta<-10^{\circ}$. 


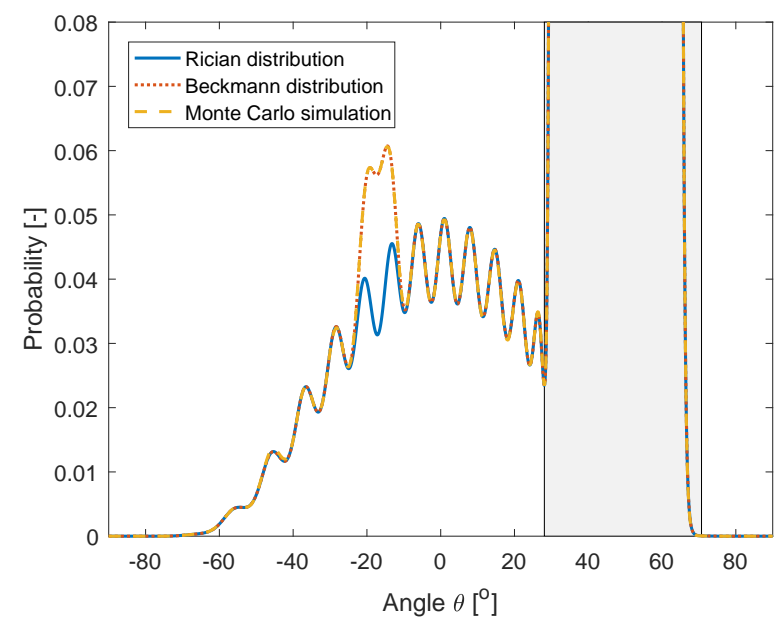

Fig. 6: Angular probability plot with $g_{n}^{2}(\theta)=\cos (\theta)$ and a steering angle of $45^{\circ}$. For this practical setting, the performance of the Rician CDF is not sufficient.

\section{PHYSICAL INTERPRETATION OF THE OBSERVED BEHAVIOR}

In the previous section, results are presented which might seem counterintuitive at first. To give a physical interpretation of this behavior, instead of only relying on the derived mathematical descriptions, a step back has to be taken. In this section, first the root cause of the largely varying variances around the angles $\theta=-90^{\circ}$, $\theta=0^{\circ}$ and $\theta=90^{\circ}$ (see Fig. 1) is presented. Next, the nonzero correlation between $X$ and $Y$ (see Fig. 2) is examined. After that, the impact of this unidentical variance and the nonzero correlation on the distribution of the magnitude of the array amplitude pattern is explained. Finally, the impact of beam steering on the angular probability plots is explained as well.

\section{A. Variance}

In order to interpret the behavior of the variance, the effect of the phase and amplitude errors are examined separately. First, the

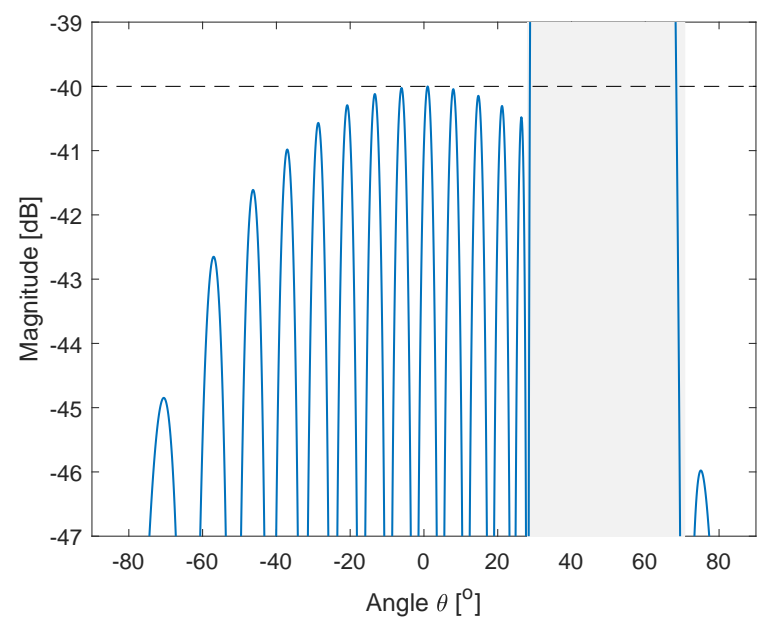

Fig. 7: Zoom of the error-free radiation pattern with $g_{n}^{2}(\theta)=\cos (\theta)$ and a steering angle of $45^{\circ}$. Note that the side lobes do not have the same magnitude.

amplitude error is assumed to be zero and only the phase is distorted. In this case, (6) reduces to

$$
F_{d}=\sum_{n=1}^{N} A_{n} e^{j \delta_{p n}} e^{j C_{n}}=\sum_{n=1}^{N} V_{n} e^{j \delta_{p n}}=\sum_{n=1}^{N} V_{n, d} .
$$

Equation (21) represents a summation of vectors in the complex plane, where $V_{n}$ and $V_{n, d}$ are the error-free and distorted vector of the $n^{\text {th }}$ element respectively. Each error-free vector $V_{n}$ is distorted by the $e^{j \delta_{p n}}$-term. This error-term only accounts for a change in angle of the vector in the complex plane, but the amplitude of the error-free vector and the distorted vector will remain the same, i.e., $\left|V_{n}\right|=\left|V_{n, d}\right|$.

In Section III, a sufficiently large $N$ was assumed to let $X$ and $Y$ approach a Gaussian distribution. However, the definitions of variance of a random variable and correlation between two random variables are independent of the underlying distribution(s), and therefore the choice of $N$ is not of high importance in the upcoming analysis. For

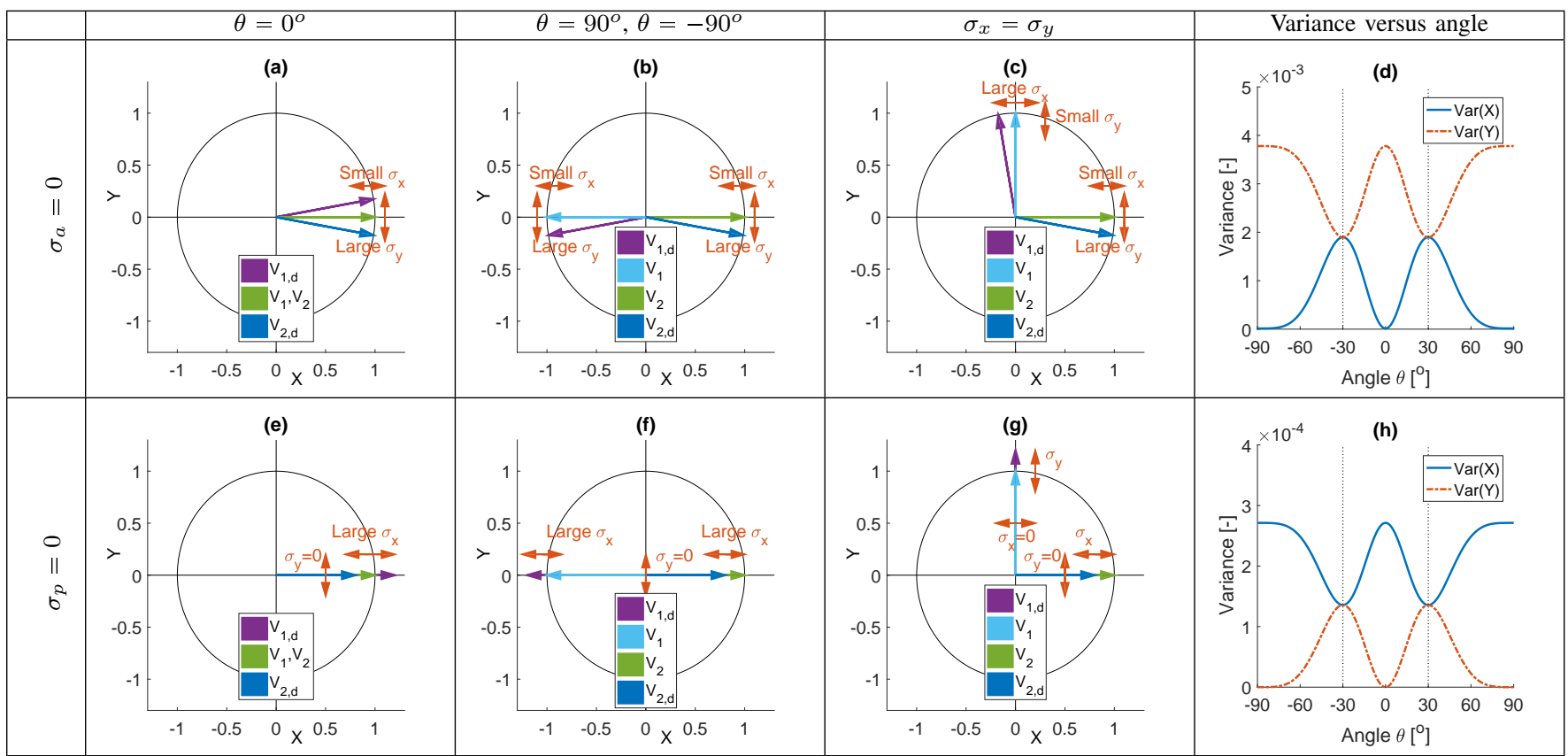

Fig. 8: Vector representation of the element excitations to explain the variance, $N=2$. 
visualization purposes, the array contains only $N=2$ elements in the upcoming analysis. Moreover, $A_{n}=1, d=\frac{1}{2} \lambda_{0}$ and no phase difference is applied amongst the elements.

For $\theta=0^{\circ}, V_{1}=V_{2}=1$, the resulting individual error-free vectors $V_{1}$ and $V_{2}$ are visualized in the unit circle in Fig. 8a. Also, one example of the distorted variants $V_{1, d}$ and $V_{2, d}$ is visualized in Fig. 8a. Here one can see that for a relatively small phase error, the resulting error in $X$ is small compared to the error in $Y$. This gives rise to a large variance in $Y$ and only a small variance in $X$.

Next, $\theta=90^{\circ}$ is considered. In this case the resulting vectors are out of phase and therefore a null is created in the error-free radiation pattern in that direction. The variance of $Y$, however, is quite large in this case. This scenario is visualized in Fig. $8 \mathrm{~b}$. Note that the scenario of $\theta=90^{\circ}$ gives rise to exactly the same variance in $X$ and $Y$ as in the case of $\theta=-90^{\circ}$ and $\theta=0^{\circ}$.

Another interesting scenario to consider is when the variance in $X$ and $Y$ are equal. It is geometrically straightforward to see in Fig. 8c that the variances in $X$ and $Y$ are equal for a $90^{\circ}$ phase difference between $V_{1}$ and $V_{2}$ in the complex plane. This corresponds to the following values of $\theta$

$$
\begin{aligned}
& \pi \sin \theta=\frac{1}{2} \pi \rightarrow \theta=30^{\circ}, \\
& \pi \sin \theta=-\frac{1}{2} \pi \rightarrow \theta=-30^{\circ} .
\end{aligned}
$$

The variances of $X$ and $Y$ for $\sigma_{a}=0 \mathrm{~dB}$ and $\sigma_{p}=5^{\circ}$ are calculated according to (10) and (11), and visualized in Fig. 8d. Note that for the special cases which are visualized in Fig. 8a-c, the graph of Fig. 8d matches the predictions.

As a second step, an amplitude error is assumed and the error in phase is omitted. In this case, (6) reduces to

$$
F_{d}=\sum_{n=1}^{N} A_{n}\left(1+\delta_{a n}\right) e^{j C_{n}}=\sum_{n=1}^{N} V_{n}\left(1+\delta_{a n}\right)=\sum_{n=1}^{N} V_{n, d}
$$

Again, (23) represents a summation of vectors in the complex plane. However, the error term now only counts for a change in magnitude, rather than changing the angle of the vector.

The resulting individual error-free vectors $V_{1}$ and $V_{2}$ for $\theta=0^{\circ}$ are visualized in the unit circle in Fig. 8e. One example of the distorted variants $V_{1, d}$ and $V_{2, d}$ is also visualized in Fig. 8e. It can be observed that for an amplitude error, the resulting error in $X$ is large, whereas the error in $Y$ is zero. This gives rise to a large variance in $X$ and zero variance in $Y$.

In Fig. 8f, $\theta=90^{\circ}$ is considered. The variance of $X$ is in this case quite large, whereas the variance in $Y$ is again zero. Also here, note that the scenario of $\theta=90^{\circ}$ gives rise to exactly the same variance in $X$ and $Y$ as in the case of $\theta=-90^{\circ}$ and $\theta=0^{\circ}$.

Figure 8g illustrates the scenario where the variance in $X$ and $Y$ are equal. As calculated in (22), this scenario corresponds to $\theta=30^{\circ}$ and $\theta=-30^{\circ}$.

The variances of $X$ and $Y$ for $\sigma_{a}=0.1 \mathrm{~dB}$ and $\sigma_{p}=0^{\circ}$ are calculated according to (10) and (11), and visualized in Fig. 8h. Note that for the special cases which are visualized in Fig. 8e-g, the graph of Fig. 8h again matches the predictions.

\section{B. Correlation}

The nonzero correlation between $X$ and $Y$ can be explained by using a similar analysis as used in explaining the behavior of the variance of $X$ and $Y$. However, in this analysis, certain regions are explored rather than looking at a few special cases.

Again, first the amplitude error is omitted. In Fig. 9a, the errorfree and distorted vectors are depicted. One can see that if an error is made in $V_{2}$, the error made in $X$ and $Y$ are uncorrelated. On the other hand, if an error has been made in $V_{1}$, the error in $X$ and $Y$ show opposite behavior. If the error in $Y$ is positive, the error in $X$ will be negative, and vice versa. Therefore, the total error in $X$ and $Y$ will have a negative correlation coefficient. Note that this behavior is present in the whole first quadrant.

If $V_{1}$ is somewhere in the second quadrant, the errors in $X$ and $Y$ show the same behavior. If the error in $Y$ is positive, the error in $X$ will also be positive, and the same for the errors being negative. This results in a positive correlation coefficient. This situation is visualized in Fig. 9b.

Next, the case for no correlation between $X$ and $Y$ is examined. As can be seen in Fig. 9c, this occurs for a $90^{\circ}$ phase difference between $V_{1}$ and $V_{2}$, which again corresponds to $\theta=30^{\circ}$ and $\theta=-30^{\circ}$.

The correlation between $X$ and $Y$ for $\sigma_{a}=0$ and $\sigma_{p}=5^{\circ}$ is calculated according to (12) and visualized in Fig. 9d. Note that for the special cases which are visualized in Fig. 9a-c, the graph of Fig. 9d matches the predictions.

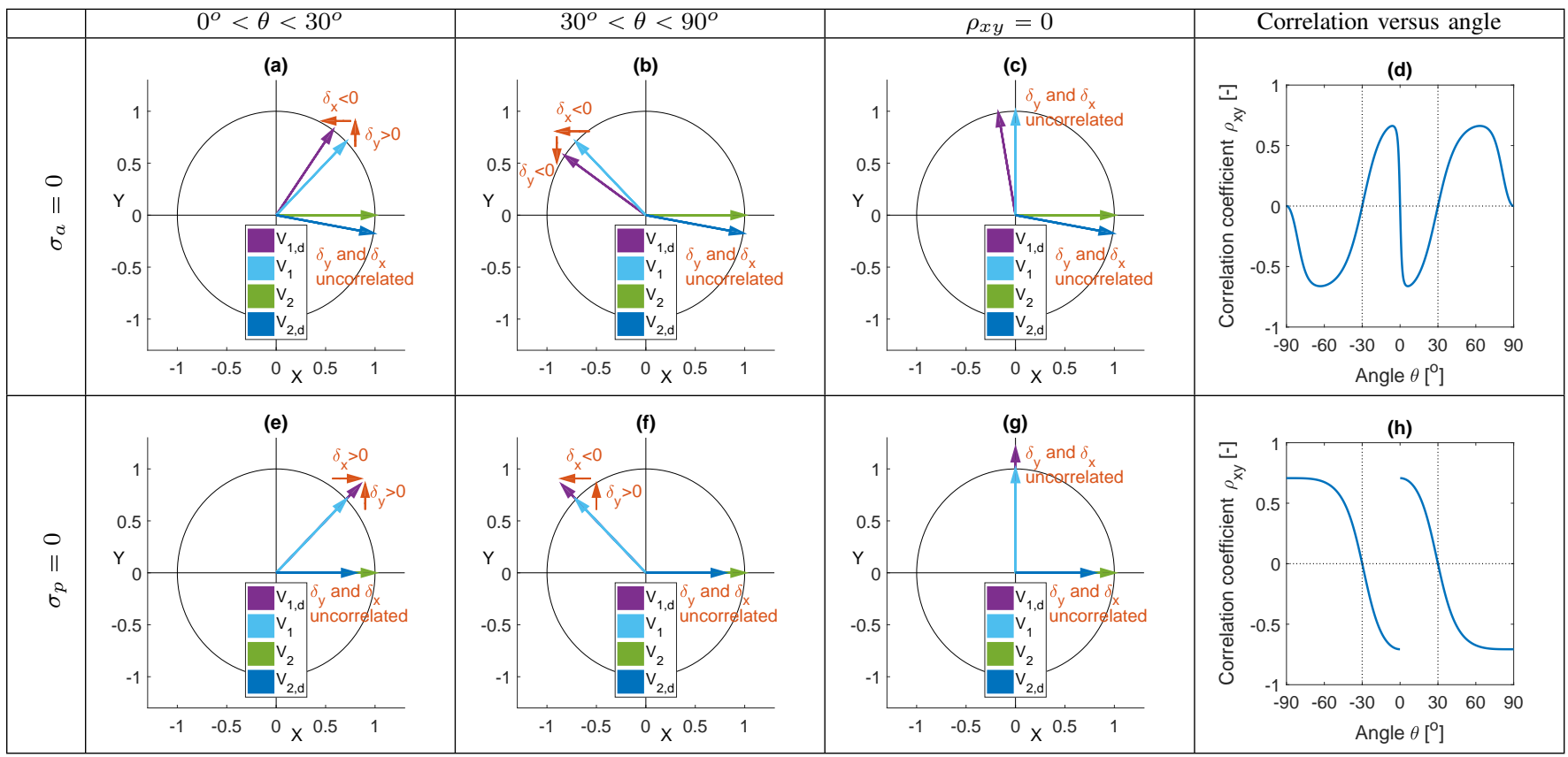

Fig. 9: Vector representation of the element excitations to explain the correlation, $N=2$. 


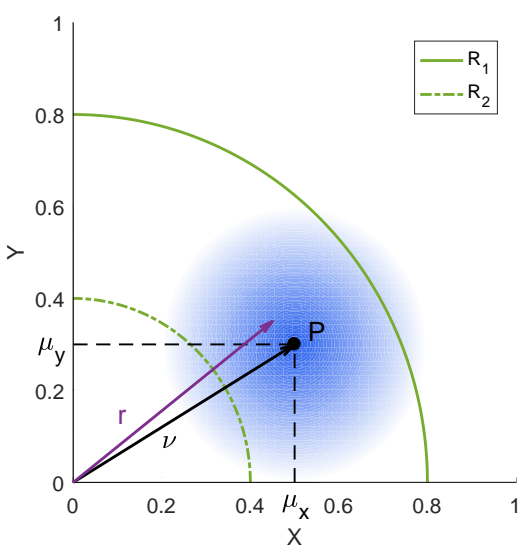

(a) Rician distribution.

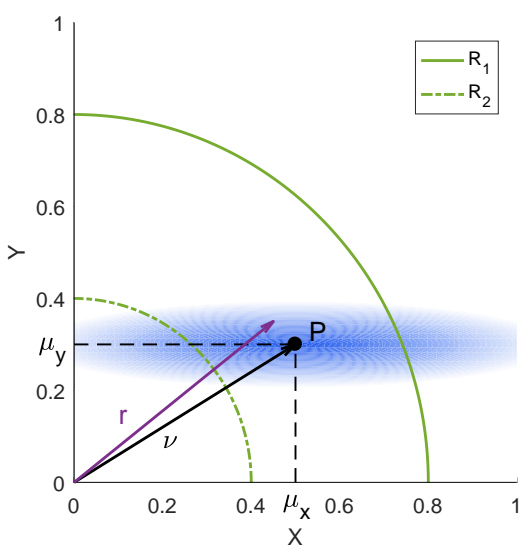

(b) Beckmann: effect of unequal variance.

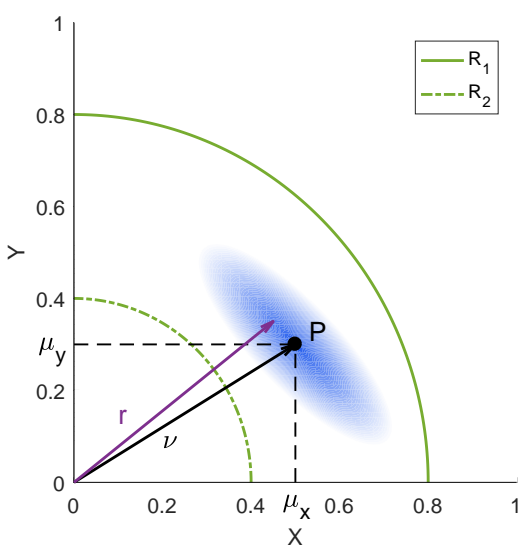

(c) Beckmann: effect of correlation.

Fig. 10: Visual representations of the Rician and Beckmann distributions.

If the phase error is omitted, one can see in Fig. 9e, that if $V_{1}$ is located in the first quadrant, the error in $X$ and $Y$ show similar behavior. If the error in $Y$ is positive, the error in $X$ will also be positive, and the same for the errors being negative. Therefore, the total error in $X$ and $Y$ will have a positive correlation coefficient.

On the other hand, if $V_{1}$ is somewhere in the second quadrant, the errors in $X$ and $Y$ show opposite behavior. If the error in $Y$ is positive, the error in $X$ will be negative, and vice versa. This results in a negative correlation coefficient. This situation is visualized in Fig. 9f.

Again, if there is a $90^{\circ}$ phase difference between $V_{1}$ and $V_{2}$, it is geometrically straightforward to see in Fig. $9 \mathrm{~g}$ that there is no correlation between $X$ and $Y$. As calculated in (22), this corresponds to $\theta=30^{\circ}$ and $\theta=-30^{\circ}$.

The correlation between $X$ and $Y$ for $\sigma_{a}=0.1 \mathrm{~dB}$ and $\sigma_{p}=0^{\circ}$ is calculated according to (12) and visualized in Fig. 9h. Note that for the special cases which are visualized in Fig. 9e-g, the graph of Fig. $9 \mathrm{~h}$ again matches the predictions.

\section{Effect on magnitude}

Previous subsections have only given a physical interpretation to the unidentical variance $\sigma_{x}^{2}$ and $\sigma_{y}^{2}$ and the correlation between $X$ and $Y$ being nonzero. The impact of this unidentical variance and nonzero correlation on the distribution of the magnitude of the array amplitude pattern is discussed here.

As mentioned in Section IV, the magnitude $r$ of the sum of two orthogonal and uncorrelated Gaussian distributions having mean $\mu_{x}$ and $\mu_{y}$ and variance $\sigma^{2}$, follows a Rician distribution. This can be graphically visualized as shown in Fig. 10a. The point $P$ represents the expected value of the array amplitude pattern for a particular angle. The parameter $\nu$ is the distance between the origin and the point $P$. Due to the variance $\sigma^{2}$, the random variables $X$ and $Y$ will take on other values than their expected values $\mu_{x}$ and $\mu_{y}$. This is illustrated using an intensity profile. The likelihood that a certain combination of $X$ and $Y$ is acquired, is represented by the intensity. If the particular position is dark blue, the likelihood of that combination of $X$ and $Y$ is high, and vice versa. Note that, since the variances for $X$ and $Y$ are equal, the intensity profile has the form of a circle.

Two quarter circles are drawn to visualize two sets of values with constant magnitude. Using the Rician CDF, the likelihood that the magnitude stays below a certain limit, e.g., the likelihood that the randomly picked points stay below one of the green curves, can be calculated. As one can see in Fig. 10a, it is, for example, in this case very likely that a randomly picked combination of $X$ and $Y$ stays below $R_{1}$, but likely to be larger than $R_{2}$.

In Fig. 10b, the effect of the unidentical variance is visualized. In this case $\sigma_{x}>\sigma_{y}$, and therefore, the shape of the blue intensity profile is changed into an ellipse. Note that this completely changes the probability of crossing a certain magnitude. Compared to the circular intensity profile of Fig. 10a, it is now more likely to cross $R_{1}$, but at the same time is has a higher probability of staying below $R_{2}$.

In Fig. 10c, the effect of correlation between $X$ and $Y$ is visualized. In this case, $\sigma_{x}=\sigma_{y}$ and $\rho_{x y}<0$. The negative correlation coefficient means that if a positive error in $X$ is made, it is more likely to make a negative error in $Y$. Therefore, due to this negative correlation, the blue intensity profile takes the shape of a 45 degrees tilted ellipse. Note that in this case, it is more likely to stay between $R_{2}$ and $R_{1}$ compared to both other cases. Again, it is clear that a nonzero correlation coefficient completely changes the probability for the magnitude of crossing a certain radiation level. ${ }^{2}$

For the parameters as specified in Section IIIb, the large variation in variance (see Fig. 1) and strong correlation (see Fig. 2) changes the distribution of $X$ and $Y$ for $|\theta|>60^{\circ}$ and around $\theta=0^{\circ}$. For $\theta=0^{\circ}$, this change in distribution is not visible for the main lobe region in Fig. 4, simply because the main lobe region will in principle always exceed the specified radiation level. At $|\theta|>60^{\circ}$, however, the probability of exceeding the specified SLL is increased compared to the rest of the side lobe region as a result of the variance and the correlation, as shown in this section. Note that, in general, for other values of $S L L_{e}$, the probability of exceeding this level can also be less compared to the rest of the side lobe region.

\section{Effect of steering}

So far, only the case of a nonzero steering angle has been analyzed. However, in practice other scan angles will be used as well. In Fig. 5, it can be observed that for a certain scan angle, the peak of higher probability shifted to a different angle. In order to understand this shift, one has to go back to the vector representations.

The large variations in the variance in $X$ and $Y$ for $\theta=-90^{\circ}$ and $\theta=90^{\circ}$ are found at the angles where $V_{1}$ and $V_{2}$ are out of phase. This essentially means that $C_{1}=\pi$ or $C_{1}=-\pi$. The linear phase distribution used in Section $\mathrm{V}$ is defined as follows

$$
\arg \left(a_{n}\right)=-k_{0} d(N-n) \sin \theta_{0},
$$

with $\theta_{0}$ the desired steering angle. By combining (3) and (24), the following expressions for $C_{1}$ can be found

$$
C_{1}=k_{0} d\left(\sin \theta-\sin \theta_{0}\right) .
$$

${ }^{2}$ Note that these examples are for illustration purposes only. It is to show the reader how unidentical variances and correlation between $X$ and $Y$ can impact the probabilities of exceeding a certain magnitude, rather than showing quantitative results. 
Solving (25) for $C_{1}=-\pi, k_{0} d=\pi$ and $\theta_{0}=45^{\circ}$ gives $\theta=$ $-17^{\circ}$. Note that this result exactly matches the behavior in Fig. 5. This physical interpretation can provide the designer knowledge about where higher probabilities of exceeding a certain radiation level are to be expected.

\section{CONCLUSION AND FUTURE WORK}

In this paper, it has been shown that the magnitude of a distorted array amplitude pattern, which is the result of random errors in the phase and amplitude in the element excitations, follows a Beckmann distribution, instead of the often assumed Rician distribution. A practical example is given where the probability of exceeding a certain radiation level is determined. In this example, it is evident that the Beckmann CDF more accurately predicts this probability compared to the Rician CDF. Moreover, the result that the maximum probability of exceeding a certain side lobe level, in general, does not have to be at the angle of the highest side lobe in the error-free case, is obtained. Due to this result, the conclusion can be drawn that angular probability plots are a necessity in properly determining the probability of exceeding a certain radiation level.

In this analysis, it is assumed that the phase and amplitude errors in the element excitations are uncorrelated. Moreover, the element patterns are assumed to be identical. In practice, however, this will not be the case. Therefore, to complete this analysis a derivation has to be performed where the random errors are correlated and the element patterns are unidentical.

\section{ACKNOWLEDGMENT}

The authors would like to thank NXP Semiconductors for their support and funding, which made this research possible.

\section{REFERENCES}

[1] J. Ruze, The Effect of Aperture Errors on the Antenna Radiation Pattern, Supplemento Nuovo Cimento 9, 364-380, 1952.

[2] J.L. Allen, Phased Array Radar Studies, Part 3, Chapter 3. Some Extensions of the Theory of Random Error Effects on Array Patterns, MIT Lincoln Laboratory Technical Report, No. 236, 1961.

[3] James K. Hsiao, Array Sidelobes, Error Tolerance, Gain and Beamdwith, NRL Report 8841, Naval Research Laboratory, 1984.

[4] J.L. Allen, The Theory of Array Antennas, Emphasis on Radar Applications, MIT Lincoln Laboratory Technical Report, No. 323, 1963.

[5] Robert E. Collin, Antenna Theory Part 1, Chapter 6.6, 1st ed., McGrawHill Book Company, 1969.

[6] Robert J. Mailloux, Phased Array Antenna Handbook, Chapter 7.2, 2nd ed., Artech House, 2005.

[7] Robert C. Hansen, Phased Array Antennas, Chapter 15.4, 2nd ed., John Wiley \& Sons, 2009.

[8] (2017, July) Marcum Q-Function - Wolfram MathWorld [Online]. Available: http://mathworld.wolfram.com/MarcumQ-Function.html

[9] P. Beckmann - Statistical Distribution of the Amplitude and Phase of a Multiply Scattered Field, Journal of Research of the National Bureau of Standards, Vol. 66D, 1961.

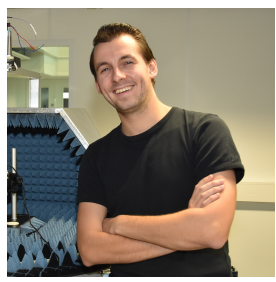

A.J. (Teun) van den Biggelaar received his M.Sc. degree in Electrical Engineering (cum laude) from the Eindhoven University of Technology (TU/e), the Netherlands, in 2016. His M.Sc. thesis was selected as the best M.Sc. thesis of the department of Electrical Engineering in the year 2016. He is currently pursuing his Ph.D. degree at the TU/e in Electrical Engineering, with the focus on calibration techniques for antenna arrays for millimeter-wave $5 \mathrm{G}$ systems.

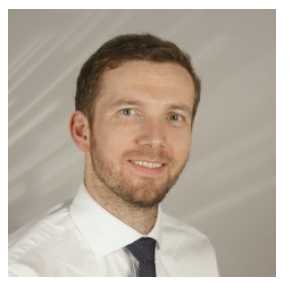

U. (Ulf) Johannsen obtained his Dipl.Ing. degree from Hamburg University of Technology (TUHH), Germany, in 2009 and his Ph.D. degree from Eindhoven University of Technology (TU/e), the Netherlands, in 2013. From 2013 until 2016 he worked as Senior Systems Engineer at ATLAS ELEKTRONIK GmbH in Bremen, Germany. His duties included the roles of system designer and engineering manager for autonomous underwater vehicle (AUV) systems with sonar payloads. Since 2016 he is assistant professor with the Electromagnetics group at the TU/e. His research focus lies on (sub-)millimeter-wave antenna systems for various applications. Moreover, he leads the Ultra-High-Data-Rate Systems programme within TU/e's Centre for Wireless Technology and he is the chair person of the IEEE Benelux AP/MTT joint chapter.

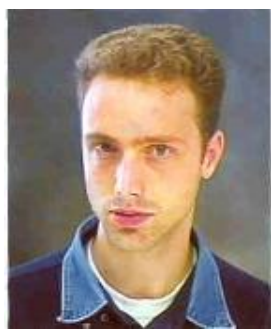

P. (Paul) Mattheijssen was born in Heerlen, the Netherlands in 1972. He received the M.Sc. degree in Electrical Engineering from Eindhoven University of Technology, Eindhoven (TU/e), the Netherlands, in 2000. From 2000 until 207 he worked as Research Scientist within Philips Research Laboratories, Eindhoven. His primary research interests include wireless communications, wave propagation, antenna design, and digital signal processing. Since 2007, he works as Senior Principal \& System Architect at NXP Semiconductors for $4 \mathrm{G}$ and $5 \mathrm{G}$ communication systems.

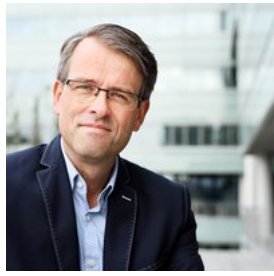

A.B. (Bart) Smolders was born in Hilvarenbeek, the Netherlands in 1965 . He received his M.Sc. and Ph.D. degree in Electrical Engineering from the Eindhoven University of Technology (TU/e) in 1989 and 1994, respectively. From 1989 to 1991, he worked as an IC Designer at FEL-TNO, The Hague. From 1994 to 1997, he was a Radar System Designer with Thales, the Netherlands. From 1997 to 2000, he was project leader of the Square Kilometer Array (SKA) with the Netherlands Foundation for Research in Astronomy (ASTRON). From 2000 to 2010, he has been with NXP (formerly Philips) Semiconductors, The Netherlands, responsible for the innovation in the RF business line. Since 2010, he is a full-time professor at the TU/e in the Electromagnetics Group with special interest in antenna systems and applications. He is junior-past chairman of the IEEE Benelux section and past-chair of the NERG (Nederlands Radio- en Elektronica Genootschap). Next to his research activities, he is the dean of the Electrical Engineering department of the TU/e. Publications can be found on: https://www.tue.nl/en/university/departments/electricalengineering/department/staff/detail/ep/e/d/ep-uid/19910474/ep-tab/4/ 